\title{
Automatic Classification of Anuran Sounds Using Convolutional Neural Networks
}

\author{
Juan Colonna \\ Universidade Federal do \\ Amazonas \\ Manaus, Brazil \\ jcolonna@inesctec.pt \\ Alípio M. Jorge \\ Universidade do Porto \\ Porto, Portugal \\ amjorge@fc.up.pt
}

\author{
Tanel Peet \\ Estonian Information \\ Technology College \\ Tallinn, Estonia \\ tpeet@itcollege.ee \\ Elsa Ferreira Gomes \\ Instituto Superior de \\ Engenharia do Porto \\ Porto, Portugal \\ efg@isep.ipp.pt
}

\author{
Carlos Abreu Ferreira \\ Instituto Politecnico do Porto \\ Porto, Portugal \\ cgf@isep.ipp.pt \\ João Gama \\ Universidade do Porto \\ Porto, Portugal \\ joao.jgama@gmail.com
}

\begin{abstract}
Anurans (frogs or toads) are closely related to the ecosystem and they are commonly used by biologists as early indicators of ecological stress. Automatic classification of anurans, by processing their calls, helps biologists analyze the activity of anurans on larger scale. Wireless Sensor Networks (WSNs) can be used for gathering data automatically over a large area. WSNs usually set restrictions on computing and transmission power for extending the network's lifetime. Deep Learning algorithms have gathered a lot of popularity in recent years, especially in the field of image recognition. Being an eager learner, a trained Deep Learning model does not need a lot of computing power and could be used in hardware with limited resources. This paper investigates the possibility of using Convolutional Neural Networks with Mel-Frequency Cepstral Coefficients (MFCCs) as input for the task of classifying anuran sounds.
\end{abstract}

\section{CCS Concepts}

-Computing methodologies $\rightarrow$ Neural networks;

\section{Keywords}

Convolutional Neural Networks; Machine Learning; MFCC; Wireless Sensor Networks; Anurans

\section{INTRODUCTION}

Amphibian populations are directly affected by environmental changes and therefore are closely related to ecosystem [4]. In addition, it is shown that there is a clear relationship between climate change and mortality in amphibian populations [3]. Therefore it can be concluded that anoma-

Permission to make digital or hard copies of all or part of this work for personal or classroom use is granted without fee provided that copies are not made or distributed for profit or commercial advantage and that copies bear this notice and the full citation on the first page. Copyrights for components of this work owned by others than tion on the first page. Copyrights for components of this work owned by others than
ACM must be honored. Abstracting with credit is permitted. To copy otherwise, or republish, to post on servers or to redistribute to lists, requires prior specific permission and/or a fee. Request permissions from permissions@acm.org.

C3S2E '16, July 20-22, 2016, Porto, Portugal

(c) 2016 ACM. ISBN 978-1-4503-4075-5/16/07 . \$ $\$ 15.00$

DOI: http://dx.doi.org/10.1145/2948992.2949016 lies in their behavior can be used as early indicators of ecological stress. Amphibian monitoring systems may help to estimate long-term changes in amphibian populations and determine the causes of changes.

Anurans belong to amphibians class and produce sounds (calls), which contain enough information to identify each species [12]. A human expert can be used to classify anurans by their calls, but this approach is slow and error-prone. One of the alternative approaches is to use Wireless Sensor Networks (WSNs) with automatic classification methods.

WSNs consist of low cost nodes which could be spread all over the desired areas [1]. This offers a good solution for automatically monitoring anurans over a large area, but low cost hardware also sets restrictions on computing power [22]. The cost of energy in data processing is much less compared to data communication. Therefore, local data processing is good option for minimizing power consumption [1]. Depending on the setup of the WSN and the complexity of the classification model, it may be more efficient to do the classification in low-level nodes and transmit only the results of classification to the sink nodes.

Deep Learning algorithms are eager learners, which means that the model can be trained on fast computers and the trained model could be used in WSN nodes with less computing power and therefore less power consumption [16]. Deep Learning has shown promising results in different research fields, being especially popular in image recognition tasks.

Studies in sound processing show that Mel-Frequency Cepstral Coefficients (MFCCs) are one of the most popular features for presenting audio signals in speech recognition [14] and music information retrieval tasks [13]. MFCCs mimic some parts of the human speech production and perception, including logarithmic perception of loudness and pitch of human auditory system [19]. Anuran call consist of short sounds, called syllables, which are a single blow of air from the lung of the anuran [12]. These syllables can be compared to phonemes in human speech and therefore MFCC features have shown good results in anuran classification tasks [5]. In the process of MFCC extraction, the signal is divided into fixed length frames and a user defined amount of MFCC features are extracted for every frame [19].

This paper investigates the possibility of using Convolu- 


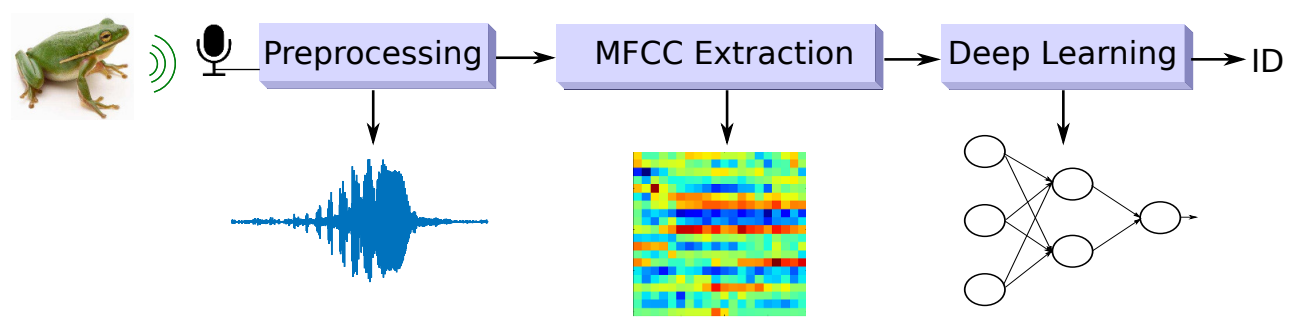

Figure 1: Anuran classification stages

tional Artificial Neural Network algorithms from image processing research for classifying anurans by their calls. In the first step, the syllables from audio signal are extracted MFCC features are extracted as a second step. The parameters are chosen in a way that after MFCC extraction, each syllable is represented by a square matrix, containing the equal amount of frames and MFCC features. The third step is using a simple Convolutional Neural Network (CNN) for classification. The approach could be seen in Figure 1. The results from the CNN are compared with different classifiers, such as k-Nearest Neighbors (kNN), Decision Tree (DT), Quadratic Discriminant Analysis (QDA) and Support Vector Machine (SVM).

The current state of the art in the field of automatic classification in bioacoustics is described in Section 2. The basic ideas and methods, which are used for training the CNN in our experiments, are explained in Section 3. Section 4 describes and analyses the experiments in more detail.

\section{STATE OF THE ART}

The approach for classifying one anuran species is done by $\mathrm{Hu}$ et. al. [11] and Taylor et. al [9]. In their proposal, all audio recording are collected and transmitted to a central node, where spectrogram is generated. The C4.5 Decision tree classifier is then used based on power features of the spectrogram. This approach requires a lot of resources due to the audio recordings which are sent to a central node.

WSNs are used for monitoring the habitats of the birds by Cai et. al. [2]. A noise reduction algorithm is proposed, based on minimum mean square error. MFCC and MFCC delta features were used for each frame of the bird calls Context Neural Network was used for classification, which uses frames from "future" and "past", taking into account the dynamic nature of the bird songs.

Vaca-Castao and Rodriguez [21] used MFCCs to classify birds and anurans with k-Nearest Neighbors classifier. The Principal Component Analysis was used in their approach for dimensionality reduction, which increased the computational cost of the whole process.

Yen and Fu [23] classify anuran species by using the results of Discrete Wavelet Transform as features. All samples were then classified by Multilayer Perceptron. Huang et. al. [12] used $\mathrm{kNN}$ and SVM classifiers to automatically recognize frog species.

Potamitis [20] and Oliveira et. al. [8] used spectrograms of bird call recordings as images for classifying the bird species. Unlike the methodology proposed in this paper, they did not segment the raw audio into syllables. Instead they turned audio signal into spectrogram and used morphological filtering to find patterns from spectrograms, which correspond to the calls of the birds. Potamitis extracted region of interests (ROIs) and used a random forest under the multi-label formulation of one vs. all for classification. Oliveira et. al algorithm extracts only the frames of the spectrogram which correspond to one certain bird species.

\section{METHODOLOGY}

The main ideas behind the methods used for classifying the anuran sounds using Convolutional Neural Networks are explained in this chapter. Signal processing and the algorithm for audio signal segmentation is described in Section 3.1. In Section 3.2, the extraction of Mel-Frequency Cepstral Coefficients (MFCCs) from audio segments is explained in detail. Section 3.3 describes the principles and building blocks of Convolutional Neural Networks.

\subsection{Preprocessing}

Pre-processing of the anuran calls used in this paper follows the steps described by Colonna et. al. [5]. First step is to standardize the signal, which includes normalization of the signal and making it have zero mean. Signal is padded with zeros with the width equal to the length of the half segment - $\beta$. After standardization and zero padding, the signal is segmented into syllables. The segmentation is done by the following algorithm, where $\alpha$ is the threshold for the normalized signal (ranging between 0 and 1 ) and $\beta$ is half of the length of the segment:

1. Find the maximum absolute value $S(t)$ of the signal;

2. If $S(t)<\alpha$ go to step 5 ;

3. Select $\beta$ seconds to the right and to the left of the peak, which gives us one syllable;

4. Extract the syllable from step 3 and replace the values in the signal with randomized small numbers to simulate noise. Go to step 1;

5. End.

The syllable extraction is illustrated in Figure 2.

\subsection{MFCC extraction}

For the experiments in this paper, the features extracted from the audio signal are Mel-Frequency Cepstral Coefficients (MFCCs), which are engineered for human speech recognition tasks and contain information about timbral features [19]. For the extraction of MFCCs, the audio signal 


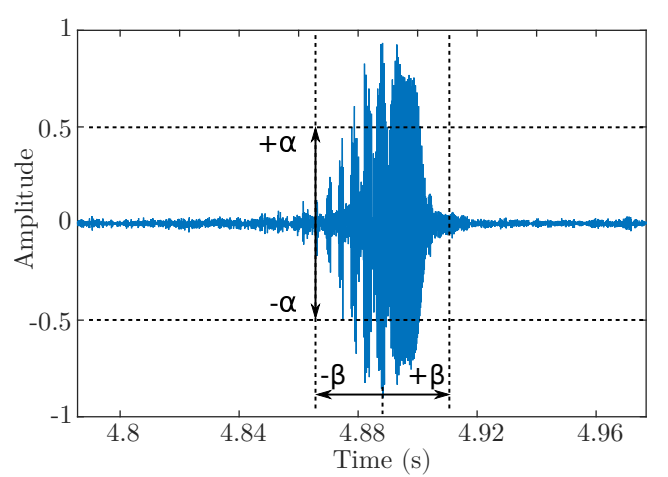

Figure 2: Syllable extraction from a Hyla Minuta species recording

is divided into short frames. For each frame the Fourier transform is taken, resulting in the spectrum of the frame. The spectrum is mapped onto the mel scale. The logs of the mel frequency spectrum are taken, followed by discrete cosine transform. The amplitudes in the resulting spectrum are MFCCs [6].

The extraction of MFCCs divides signal into multiple frames, which size is called window length. The division of audio signal to MFCC frames can be seen in Figure 3. The parameter named window step shows how much the window moves before calculating the features for the next frame, meaning the frames can overlap each other. For each frame an user defined amount of features are calculated, usually between 8 and 14 for optimal amount of information [15].

\subsection{Convolutional Neural Network}

Convolutional Neural Networks (CNNs) are variants of Multilayer Perceptron, inspired by biology and by animal visual cortex. The visual cortex of an animal contains a complex arrangement of cells which are sensitive to small regions of the visual field. These small regions are tiled to cover the entire visual area [18]. The similar approach is used in CNNs.

The layers in a CNN have neurons arranged in 3 dimensions: width, height and depth. In convolution layer the neurons are scanned by moving a fixed width, length and depth sized selection, called kernel, over the width and depth dimensions of the layer. The amount of displacement of the kernel after each step is called stride. The output of each kernel has a user defined depth and a unit width and length. The behavior in the edges of the input data is defined by zero-padding parameter. Pooling layers are often used between convolutional layers for controlling overfitting. The last layer of convolutional layer is usually fully connected layer, meaning that neurons in a fully connected layer have full connections to all activations in the previous layer [18]. The structure of the CNN used in the experiment is shown on Figure 4.

\section{EXPERIMENT}

The following chapter describes the setup and results of the experiments on anuran sound classification. Section 4.1 explains how the dataset is defined and built. Two binary classification problems are defined in Section 4.2. The con-

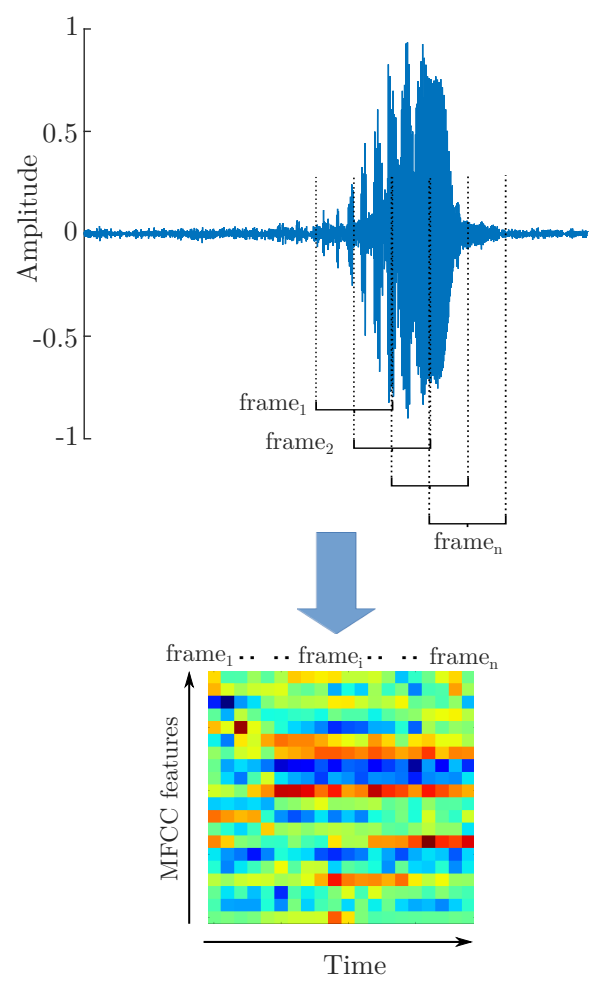

Figure 3: MFCC extraction from a Hyla Minuta syllable

figuration for MFCC extraction, different Machine Learning approaches for anuran classification and the evaluation of results is described in Section 4.3. The results of the two experiments can be found in Section 4.4, followed by the analysis of these results in Section 4.5.

\subsection{Dataset}

The dataset used in our experiments contains ten anuran species from four different families. The sound recordings were collected in the anuran habitat, under real noise conditions. The recordings for Ameerega Trivittata, Hyla Minuta, Scinax Ruber and Adenomera Hylaedactyla were extracted from three different regions: Mata Atlantica, Brazil [10], Bolivia [7] and French Guiana [17]. The rest of the recordings were collected on the campus of the Federal University of Amazonas in Manaus, Brazil [5]. Species used in our experiments can be seen in Table 1 .

The recordings in the dataset have different length and therefore contain a different number of syllables. The number of syllables extracted from one recording varies from 3 syllables (Osteocephalus Oophagus recording) to 1071 syllables (Rhinella Granulosa recording). The recordings have different kind of background noises, including natural ones but also noises specific to the recording device and its settings. Hence, it is important to separate training and dataset by recordings, not by syllables. The audio recordings are stored in wav format with a sample rate of $44.1 \mathrm{kHz}$ and 32 bits per sample.

In the preprocessing phase the value of $\alpha$ was 0.5 and $\beta$ was 0.101 seconds. The results of the syllables extraction 
Input

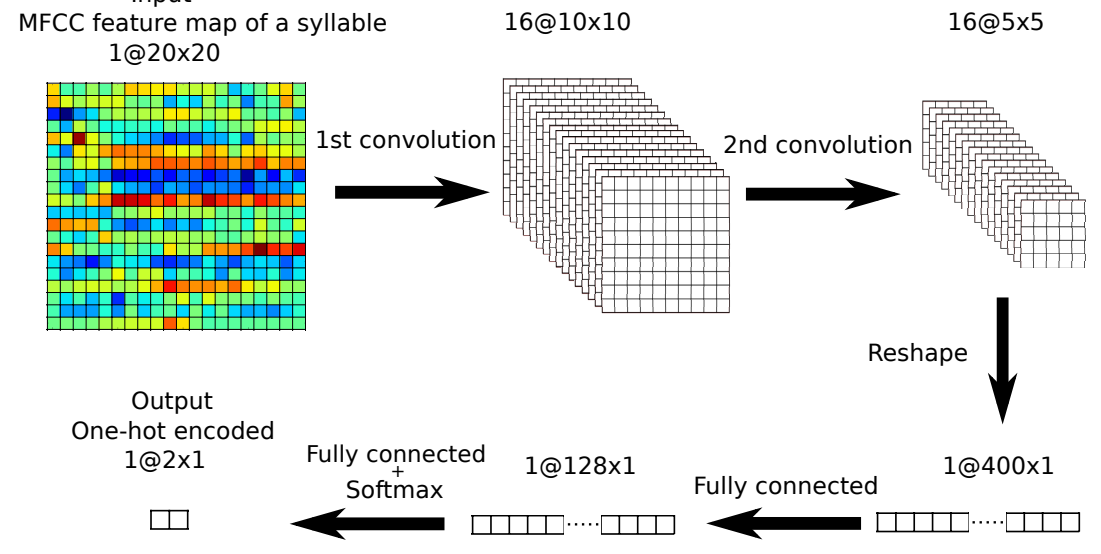

Figure 4: Structure of the CNN used in experiments

can be seen in Table 1. In addition to species label, each syllable has also a label containing the record identifier to distinguish from which recording the syllable was extracted. Each syllable has a length of $202 \mathrm{~ms}$.

\subsection{Problem}

Two different binary classification experiments are performed using Deep Learning algorithms. The obtained results are compared against five other classifiers. In the first experiment the Leptodactylidae family of anurans forms one class and all the other families form the second class. The Leptodactylidae class consists of Adenomera Andreae, Adenomera Hylaedactyla and Leptodactylus Fuscus syllables. The other families class consists of the syllables from Ameerega Trivittata, Hyla Minuta, Hypsiboas Cinerascens, Hypsiboas Cordobae, Osteocephalus Oophagus, Scinax Ruber and Rhinella Granulosa. The Leptodactylidae family class has 3812 syllables from 23 recordings and other families class has 3972 syllables from 32 recordings.

The goal of the second experiment is to recognize one species from Leptodactylidae family - Adenomera Hylaedactyla Hence, it is a binary classification where Adenomera Hylaedactyla species forms one class and all the other species form the second one. The other species class contains therefore all the syllables from Adenomera Andreae, Leptodactylus Fuscus, Ameerega Trivittata, Hyla Minuta, Hypsiboas Cinerascens, Hypsiboas Cordobae, Osteocephalus Oophagus, Scinax Ruber and Rhinella Granulosa species. The Adenomera Hylaedactyla class has 3084 syllables from 11 recordings compared to other species class with 4700 syllables from 44 recordings.

\subsection{Configuration of the experiments}

After the preprocessing phase the MFCCs are extracted for each syllable. The experiments done with Convolutional Neural Network (CNN) use MFCCs with 20 features. The window length and window step size are chosen to get 20 frames for each syllable, resulting in 20x20 matrix. The values for window length and window step in MFCC extraction are accordingly $20 \mathrm{~ms}$ and $10 \mathrm{~ms}$. For all the other classifiers, the window length and step size are the same as the length of a syllable. This results in a 20 dimensional vector for each syllable.
The Convolutional Neural Network has two convolutional layers, followed by two fully connected layer, as described in Figure 4. Rectified Linear Units (ReLUs) were used as an activation function for each convolutional layer for increasing the nonlinear properties of the network. No pooling layers were used. The output layer is one-hot encoded array with two elements, where each element represents the probability of the corresponding class.

The kernel size of $3 \times 3$ is used with a stride of 2 . The depth of the convolutional layers is 16 . The number of neurons in the fully connected hidden layer is 128. Stochastic gradient descent was used for training with a learning rate of 0.05. The training takes 200 steps, each using a batch of 16 syllables.

Leave-one-out cross-validation (LOOCV) is used on recordings for measuring the performance of the classification algorithms. The syllables are divided into two classes based on the definition of the problem. Each step of the LOOCV takes all the syllables from one recording and separates them from the training set for later testing. These steps are repeated until every recording has been used as a test set. In every step, the predictions for every syllable are saved. After LOOCV is completed, accuracy is calculated by dividing the number of correctly predicted syllables by the total number of syllables. In addition, F1 score and area under receiver operating characteristic curve (AUC) are used with the predictions from LOOCV as an performance measures of the algorithms.

The results from the CNN are compared to the results of different classification algorithms, such as k-Nearest Neighbors (kNN), Decision Tree (DT), Quadratic Discriminant Analysis (QDA) and Support Vector Machine (SVM). The $\mathrm{k}$ of $\mathrm{kNN}$ was set to 50 , which yielded the best results, compared to other values of $\mathrm{k}$. A radial basis function kernel was used in SVM training. QDA and Decision Trees were used with their default settings. ${ }^{1}$

Python was used for segmentation, MFCC extraction and CNN. Scikit-learn ${ }^{2}$ library was used for several data processing tasks. MFCC feature extraction was done by the help of

\footnotetext{
${ }^{1}$ fitcknn(), fitctree(), fitcdiscr() and fitcsvm() functions in Matlab were used

${ }^{2}$ http://scikit-learn.org/
} 
Table 1: The dataset description containing species of anurans grouped by their families

\begin{tabular}{cccc}
\hline \hline Family & Species & Recordings & Syllables \\
\hline \hline Leptodactylidae & & $\mathbf{2 3}$ & $\mathbf{3 8 1 2}$ \\
& Adenomera Andreae & 8 & 487 \\
& Adenomera Hylaedactyla & 11 & 3084 \\
& Leptodactylus Fuscus & 4 & 241 \\
\hline Dendrobatidae & & $\mathbf{5}$ & $\mathbf{3 6 6}$ \\
& Ameerega Trivittata & 5 & 366 \\
\hline Hylidae & Hyla Minuta & $\mathbf{2 4}$ & $\mathbf{2 0 3 7}$ \\
& Hypsiboas Cinerascens & 2 & 241 \\
& Hypsiboas Cordobae & 4 & 343 \\
& Osteocephalus Oophagus & 3 & 1168 \\
& Scinax Ruber & 4 & 103 \\
\hline Bufonidae & Rhinella Granulosa & 3 & 1569 \\
\hline \hline Total & $\mathbf{3}$ & $\mathbf{7 7 8 4}$ \\
\hline \hline
\end{tabular}

python_speech_features library ${ }^{3}$. For the creation of CNN the Tensorflow ${ }^{4}$ library was used. The classification with other classifiers was done in Matlab ${ }^{5}$.

\subsection{Results}

The results for evaluation of anuran classification algorithms can be seen in Table 2. Problem $\mathbf{I}$ is the classification of whether the anuran call is from Leptodactylidae family or not. The second problem II is the classification of whether the anuran call is from Adenomera Hylaedactyla species or not.

\subsection{Analysis of results}

Convolutional Neural Networks outperformed other classifiers in both problems and all the evaluation metrics, as seen in Table 2.

To get a better understanding of what the CNN was able to learn, the results were analyzed by looking at the performance of each recording in LOOCV steps. For the first problem, where anurans from Leptodactylidae family were being identified, the accuracy for each species identification was calculated for the purpose of further analysis. The identification accuracy for Adenomera Andreae, Adenomera Hylaedactyla and Leptodactylus Fuscus was $66.55 \%, 99.17 \%$ and $40.40 \%$ accordingly. Correlation can be seen between the number of syllables for a species and their identification accuracy. This means that due to the larger amount of training data available for the Adenomera Hylaedactyla species (3084 syllables), the algorithm learns to recognize this species a lot better than other members of the Leptodactylidae family - Leptodactylus Fuscus (241 syllables) and Adenomera Andreae (487 syllables).

\section{CONCLUSIONS}

In this study, the usage of Deep Learning image classification algorithms in anuran sounds classification task was proposed for the usage in Wireless Sensor Networks. The approach included the segmentation of the audio recording

${ }^{3}$ http://python-speech-features.readthedocs.org/en/latest/

${ }^{4}$ https://www.tensorflow.org/

${ }^{5}$ http://www.mathworks.com/products/matlab/ to syllables and extracting suitable sized MFCC features, followed by Deep Learning classification algorithm. The performance of the model was evaluated and compared to other classifiers. The proposed approach outperformed all the other classifiers, achieving $91.41 \%$ accuracy in binary classification task of identifying Leptodactylidae family members and $99.14 \%$ accuracy in binary classification for identifying the members of Adenomera Hylaedactyla species.

The future work will investigate the usage of different algorithms and hyper-parameters for increased performance. The analysis of results showed that the unbalanced dataset might be problematic for identifying some species and that results could be improved by collecting more training data. The usage in WSNs should also be validated in future works by comparing the cost of classification and cost of transmitting one number instead of MFCC features.

\section{ACKNOWLEDGMENTS}

This work was financed by the ERDF - European Regional Development Fund through the Operational Programme for Competitiveness and Internationalisation - COMPETE 2020 Programme within project POCI-01-0145-FEDER-006961, and by National Funds through the FCT - Fundação para a Ciência e a Tecnologia (Portuguese Foundation for Science and Technology) as part of project UID/EEA/50014/2013.

Authors JC and JG acknowledge that in addition, the project was partly supported by the European Commission through MAESTRA (ICT-2013-612944).

\section{REFERENCES}

[1] I. F. Akyildiz, W. Su, Y. Sankarasubramaniam, and E. Cayirci. Wireless sensor networks: a survey. Computer networks, 38(4):393-422, 2002.

[2] J. Cai, D. Ee, B. Pham, P. Roe, and J. Zhang. Sensor network for the monitoring of ecosystem: Bird species recognition. In Intelligent Sensors, Sensor Networks and Information, 200\%. ISSNIP 2007. 3rd International Conference on, pages 293-298. IEEE, 2007.

[3] C. Carey, W. R. Heyer, J. Wilkinson, R. A. Alford, J. W. Arntzen, T. Halliday, L. Hungerford, K. R. Lips, 
Table 2: The comparison of different classification algorithm results. Problem I - classifying Leptodactylidae family; II - classifying Adenomera Hylaedactyla species

\begin{tabular}{ccccccc}
\hline Prob. & Metric & kNN & DT & QDA & SVM & CNN \\
\hline \multirow{4}{*}{ I } & Accuracy & 0.890 & 0.863 & 0.909 & 0.901 & $\mathbf{0 . 9 1 4}$ \\
& F1 Score & 0.886 & 0.854 & 0.905 & 0.892 & $\mathbf{0 . 9 2 1}$ \\
& AUC & 0.964 & 0.880 & 0.941 & 0.962 & $\mathbf{0 . 9 7 0}$ \\
\hline \multirow{2}{*}{ II } & Accuracy & 0.939 & 0.931 & 0.950 & 0.964 & $\mathbf{0 . 9 9 1}$ \\
& F1 Score & 0.926 & 0.912 & 0.937 & 0.954 & $\mathbf{0 . 9 9 4}$ \\
& AUC & 0.988 & 0.945 & 0.980 & 0.990 & $\mathbf{0 . 9 9 9}$
\end{tabular}

E. M. Middleton, S. A. Orchard, and A. S. Rand. Amphibian declines and environmental change: Use of remote-sensing data to identify environmental correlates. Conservation Biology, 15(4):903-913, 2001.

[4] J. P. Collins and A. Storfer. Global amphibian declines: sorting the hypotheses. Diversity and distributions, 9(2):89-98, 2003.

[5] J. G. Colonna, A. D. Ribas, E. M. dos Santos, and E. F. Nakamura. Feature subset selection for automatically classifying anuran calls using sensor networks. In Neural Networks (IJCNN), The 2012 International Joint Conference on, pages 1-8, June 2012.

[6] S. B. Davis and P. Mermelstein. Comparison of parametric representations for monosyllabic word recognition in continuously spoken sentences. Acoustics, Speech and Signal Processing, IEEE Transactions on, 28(4):357-366, 1980.

[7] I. De la Riva, S. Reichle, J. Köhler, S. Lötters, J. Bosch, S. Mayer, A. Hennessey, and J. Padial. Sounds of frogs and toads of bolivia, 2002.

[8] A. G. de Oliveira, T. M. Ventura, T. D. Ganchev, J. M. de Figueiredo, O. Jahn, M. I. Marques, and K.-L. Schuchmann. Bird acoustic activity detection based on morphological filtering of the spectrogram. Applied Acoustics, 98:34 - 42, 2015.

[9] G. Grigg, A. Taylor, H. Mc Callum, and G. Watson. Monitoring frog communities: an application of machine learning. In Proceedings of Eighth Innovative Applications of Artificial Intelligence Conference, Portland Oregon, pages 1564-1569, 1996.

[10] C. Haddad, J. Giovanelli, L. Giasson, and L. Toledo. Guia sonoro dos anfíbios anuros da mata atlântica. Commercial digital media. Manaus: NovoDisc Midia Digital da Amazônia Ltda, 2005.

[11] W. Hu, N. Bulusu, C. T. Chou, S. Jha, A. Taylor, and V. N. Tran. Design and evaluation of a hybrid sensor network for cane toad monitoring. ACM Transactions on Sensor Networks (TOSN), 5(1):4, 2009.

[12] C.-J. Huang, Y.-J. Yang, D.-X. Yang, and Y.-J. Chen. Frog classification using machine learning techniques. Expert Systems with Applications, 36(2, Part 2):3737 3743, 2009.

[13] E. J. Humphrey, J. P. Bello, and Y. LeCun. Feature learning and deep architectures: new directions for music informatics. Journal of Intelligent Information Systems, 41(3):461-481, 2013.

[14] B. Logan et al. Mel frequency cepstral coefficients for music modeling. In ISMIR, 2000.
[15] R. Loughran, J. Walker, M. O'Neill, and M. O'Farrell. The use of mel-frequency cepstral coefficients in musical instrument identification. In International Computer Music Conference, Belfast, Northern Ireland. Citeseer, 2008.

[16] I. G. Maglogiannis. Emerging artificial intelligence applications in computer engineering: real word AI systems with applications in eHealth, HCI, information retrieval and pervasive technologies, volume 160. Ios Press, 2007.

[17] C. Marty and P. Gaucher. Sound guide to the tailless amphibians of French Guiana. CEBA, 1999.

[18] M. Matsugu, K. Mori, Y. Mitari, and Y. Kaneda. Subject independent facial expression recognition with robust face detection using a convolutional neural network. Neural Networks, 16(5-6):555 - 559, 2003. Advances in Neural Networks Research: IJCNN '03.

[19] P. Mermelstein. Distance measures for speech recognition, psychological and instrumental. Pattern recognition and artificial intelligence, 116:374-388, 1976.

[20] I. Potamitis. Automatic classification of a taxon-rich community recorded in the wild. PloS one, 9(5):e96936, 2014.

[21] G. Vaca-Castano and D. Rodriguez. Using syllabic mel cepstrum features and k-nearest neighbors to identify anurans and birds species. In Signal Processing Systems (SIPS), 2010 IEEE Workshop on, pages 466-471. IEEE, 2010.

[22] G. Xing, X. Wang, Y. Zhang, C. Lu, R. Pless, and C. Gill. Integrated coverage and connectivity configuration for energy conservation in sensor networks. ACM Transactions on Sensor Networks (TOSN), 1(1):36-72, 2005.

[23] G. G. Yen and Q. Fu. Automatic frog call monitoring system: a machine learning approach. In AeroSense 2002, pages 188-199. International Society for Optics and Photonics, 2002. 